CHAPTER III

THE CLASSICAL QUANTITIES OF COSMOLOGY 


\title{
THE STATUS OF THE HUBBLE DIAGRAM IN 1986
}

\author{
Hyron Spinrad \\ Astronomy Department \\ University of California \\ Berkeley, CA 94720, USA. \\ S. Djorgovski \\ Harvard-Smithsonian Center for Astrophysics \\ 60 Garden St. \\ Cambridge, MA 02138, USA.
}

\begin{abstract}
We present visual and near-infrared Hubble diagrams for firstranked cluster galaxies, and moderate-flux and high-flux radio galaxies. The photometric improvements and the extension of the diagrams to large redshifts (up to $z \simeq 1.8$ ) for both $3 \mathrm{CR}$ and " 1 Jy" class radio galaxies are highlighted. The absolute luminosities of these three types of "standard candles", where their redshifts overlap, agree adequately. The near-IR $(2 \mu \mathrm{m})$ Hubble diagrams may be used to determine the global deceleration parameter, $q_{0}$, and the current data favor $q_{0} \sim+0.2$ to +0.3 . The sensitivity to evolutionary changes in the giant galaxies is quite modest at these long wavelengths. On the other hand, the visual regime $(B V R)$ shows a dramatic dependence on differential evolution effects, which dominate over the cosmological model differences. Most radio galaxies apparently have had active star formation, with a continually declining rate, but a few are quite faint and red; they may have had a relatively passive evolution.
\end{abstract}

\section{Introduction. The New Observational Capabilities}

The principal goal of cosmology is to determine the global geometric structure and evolutionary history of the Universe. The Hubble diagram, a plot of the $(m, z)$ relation for a given type of luminous galaxies, has been one of the classic methods in this quest ( $c f$. Sandage 1975). But in the real world these goals, in particular the determination of the deceleration parameter $q_{0}$ and galaxy luminosity evolution, have been sufficiently intertwined so that neither have been determined with any certainty so far. [We will interchangeably use $2 q_{0}=\Omega_{0}$, and assume $\Lambda_{0}=0$ in this paper.] This research is difficult: we are still beset with sample selection biases that are probably functions of redshift; there remain some problems with the faint end of the galaxy photometry, and to a lesser degree, with the bright end aperture corrections. The most referenced works on the subject in the 1970's (Gunn and Oke 1975, Kristian et al. 1978) were inconclusive and somewhat contradictory in their determinations of $q_{0}$. This was at least partly due to the galaxy evolution uncertanties. Kristian et al. (1978) and Sandage (1972) found from the brightest 
cluster members (BCM's) Hubble diagrams an apparent (evolution uncorrected) value of $q_{0} \geq 1$. Application of the simple E-galaxy evolution models by Tinsley $(1972,1977)$ suggested $q_{0}($ corrected $) \simeq+0.3$ to +0.5 , again in the provocative, near-closure range.

However, because of the recent advances in the solid state detectors (CCD's) now available to the observational astronomers of the 1980's, we can probably do better. Area photometry with CCD's now permits identification and photometry of radio galaxies and optically-selected BCM's down to $V \geq 24$. This procedure is also superior to the old aperture photometry, because apertures of any size can be synthesized, unwanted companions can be excised, and, most important for good faint object photometry, the dominant contribution of the night sky foreground

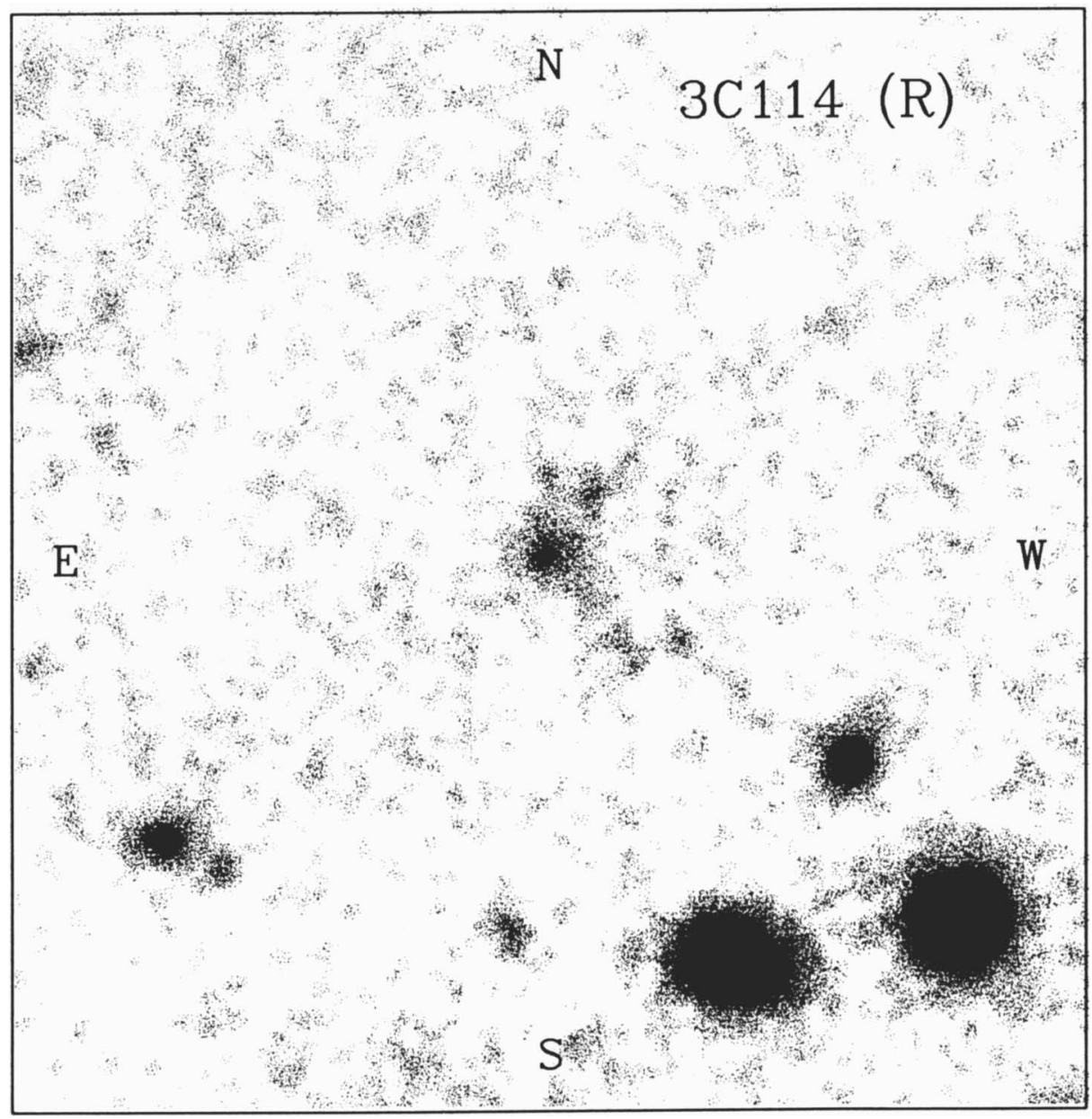

Figure 1. A 1-arcmin square section of a red CCD frame centered on 3 C114. This distant radio galaxy $(z=0.81, R=22.2)$ was identified only recently, by using a modern radio map. This image, and the one shown in Figure 2, were obtained with a TI CCD camera on the KPNO 4-m telescope in December 1985. 
can be measured with considerable precision. Figure 1 shows a field centered on $3 \mathrm{C} 114$, a $R=22.2$ radio galaxy at $z=0.815$. Figure 2 shows the field of $3 \mathrm{C} 210$, which is at $z=1.17$. Photometry of distant galaxies like these in the visual region is now fairly routine. These $3 \mathrm{CR}$ radio galaxies are quite extended even at $z>1$, and very rarely show bright or blue nuclei; they are mainly radiating starlight, even in the observed $V$ band ( emitted UV).

Our ability to measure galaxy magnitudes in the near-IR, especially in the $K$-band $(2.2 \mu \mathrm{m})$ has also steadily improved through the 1980 's. Early reviews were given by Lebofsky (1980) and Grasdalen (1980). The new data, mainly compiled by Lilly and Longair (1982, 1984; hereafter LL), Lebofsky (1981), Lebofsky and Eisenhardt (1986; hereafter LE) and Lilly et al. (1985b) are of high quality and

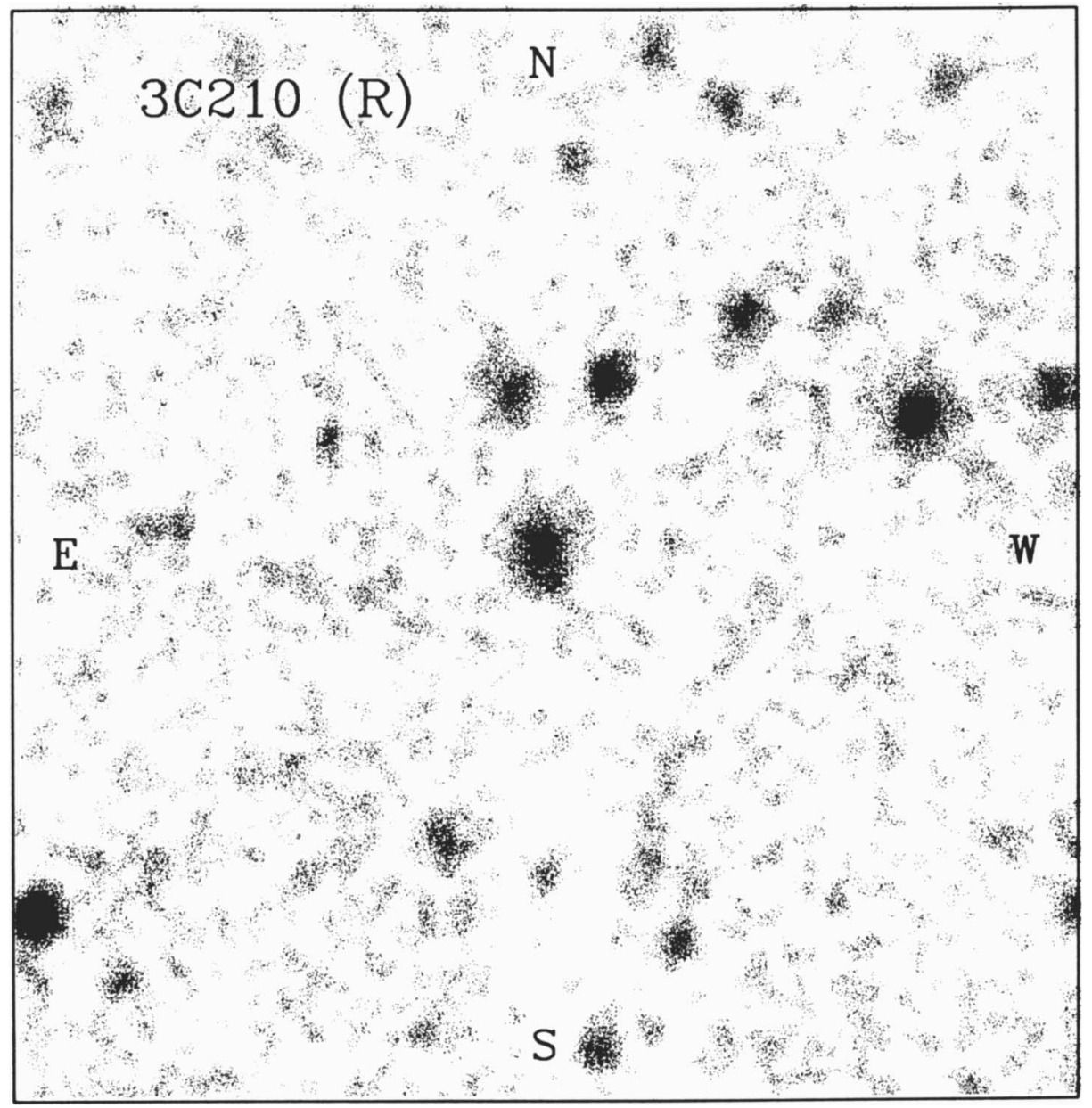

Figure 2. A red CCD frame of 3 C 210 , a faint $(R=21.5)$ radio galaxy at $z=1.17$. The field is 1 arcmin square. Note the obvious resolution of the object, and a substantial elongation in the $\mathrm{N}-\mathrm{S}$ direction. The galaxy has moderately weak emission lines indicative of a very low ionization state. 
enable us to sample the brightness of active and quiescent high-redshift galaxies near their spectral energy distribution maxima. Even in most powerful radio galaxies the stellar radiation from old stars dominates any non-thermal radiation that may be present at these long wavelengths (Lilly et al. 1985a).

Finally, and most importantly, the availability of fast CCD slit spectrographs has yielded new faint galaxy redshifts, e.g., for the Gunn, Hoessel, and Oke (1986) clusters, for 24 intermediate-power "1 Jy" galaxies (Allington-Smith et al. 1985), and for 21 powerful 3CR galaxies with $z>1$ (Spinrad and Djorgovski 1984, and in preparation). Of course, the presence of emission lines in the radio galaxy spectra greatly simplifies the measurements of their redshifts, and study of their physical properties ( $c f$. Spinrad and Djorgovski 1984, Spinrad 1986, and Perryman et al. 1984). Not all of these objects have good photometry as yet. Progressing out to $z \simeq 1.8$ for both classes of radio galaxies is critical to a new interpretation of the Hubble diagram; recall that the papers from the 1970's had available redshifts for the samples reaching only to about $z \simeq 0.5$.

Another interpretative tool of the 1980's, which we use intensively, is the set of galaxy evolution models of Bruzual (1983). These models are by intent simple, yet sufficiently predictive. With some reasonably justified assumptions on the IMF and the star formation rate (SFR) history, we can predict luminosities and colors as functions of redshift for evolving galaxies.

While these strong radio sources may not be ideal "standard candles" from all points of view, they do have one redeeming virtue: since the $3 \mathrm{CR}$ sample is now virtually complete in its identification content, and at the $97 \%$ completeness level in redshift determinations for the "safe" extragalactic identifications (Spinrad et al. 1985; Spinrad 1986), we can virtually ignore the standard statistical problem plaguing many samples, viz. the faint-end Malmquist bias. The galaxies we use are radio-selected, with no apparent correlation between the optical and the radio luminosities. Low optical luminosity might only matter for perhaps the remaining $3 \%$ of the 3CR galaxies so far unidentified, or without measured redshifts. The problem of luminosity selection is thus not very serious.

\section{Results in the Visible Regime}

Djorgovski, Spinrad, and Dickinson (1987; hereafter DSD), following the early presentation by Djorgovski, Spinrad, and Marr (1985), have used new CCD photometry of 3CR galaxies, combined with a little of spectrophotometry of slightly lower precision, to extend the visual $(B V R)$ Hubble diagrams to $z \simeq 1.82$. Only the NLRG were used (this meant discarding a few broad-line $\mathrm{N}$-galaxies, such as 3C 297, and 208.1). The DSD 3CR galaxy photometry was combined with other photometric data on the lower redshift BCM's by the various Mt. Palomar groups (Gunn and Oke 1975, Sandage et al. 1976, Kristian et al. 1978, Hoessel et al. 1980, and Schneider et al. 1983). The photometric systems were standardized with a fair success to the $B V R$ Mould-Bessel system, though it is probably true that the full DSD sample is not on a perfectly homogenous photometric system throughout the visible regime. The differential zero-point and small color terms which probably still remain are small when compared to our internal faint end photometric 
uncertainties, and are much smaller than the evolutionary effects which we discuss below. We present the photometry without the cluster richness corrections, or anything beyond the aperture corrections for $z<0.5$; the K-corrections are incorporated in the model curves.

Figure 3 illustrates the contemporary Hubble diagram for all galaxy classes in the $V$-band, with several evolutionary models overlaid. The Bruzual models shown are characterized either by an early single 1-Gyr burst of star formation ( $c$-models), or an exponentially decreasing SFR rate ( $\mu$-models; $\mu=0.5$ implies an $e$-folding time of about $1.4 \mathrm{Gyr}$ ). Also shown are the changes in models for an open $\left(\Omega_{0}=0=q_{0}\right)$ and the critical $\left(\Omega_{0}=1\right.$, or $\left.q_{0}=\frac{1}{2}\right)$ universes. A fictitious

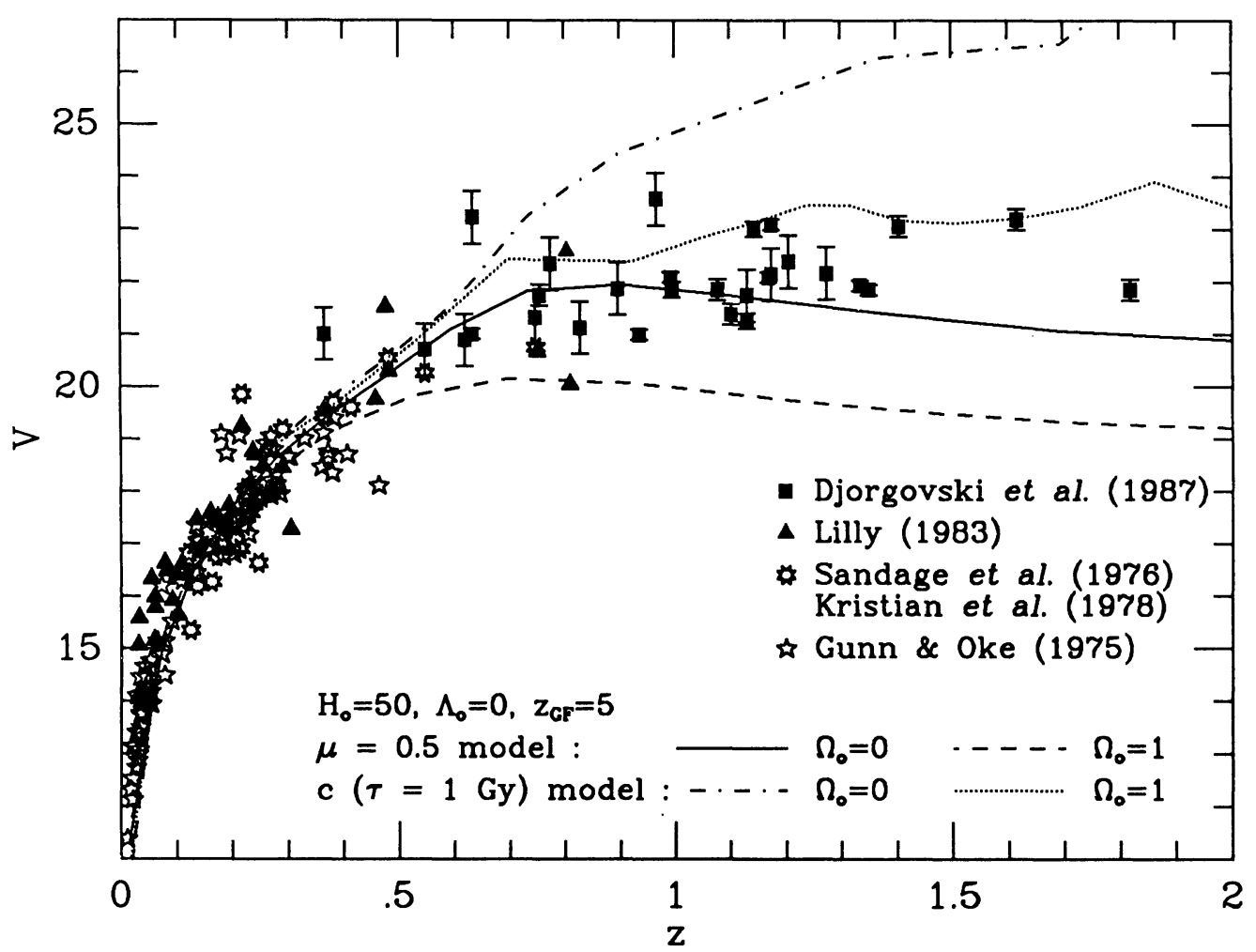

Figure 3. The visual region Hubble diagram for 3CR galaxies and BCM's. The data include cluster $\mathrm{gE}$ 's at the lower redshift $(z \leq 0.76)$, and $3 \mathrm{CR}$ radio galaxies at the higher redshifts. Superposed are the simple cosmological models described by $q_{0}=\Omega_{0}=0$ and $q_{0}=\frac{1}{2}$, combined with two galaxy evolution models by Bruzual: one is a $c$-model with a 1 Gyr burst duration, the other is for $\mu=0.5$ (exponential SFR decay, with an $e$-folding time of $\simeq 1.4 \mathrm{Gyr}$ ), both with a Salpeter IMF. Note the relatively large sensitivity to galactic evolution and the modest dependence on the cosmological model; in the $V$-band one cannot satisfactorily separate the two effects. A non-evolving model (just a K-corrected present-day E-galaxy energy distribution) at $z \geq 1.5$ lies $\sim 2^{m}$ above the upper $c$-model curve, and $\sim 5^{m}-6^{m}$ above the $\mu$-model curves and the data. 
"No evolution" model (not shown), incorporating the K-correction alone would be consistently fainter than the data at the high redshifts. The effect of the global geometry is considerably smaller than the range of evolutionary changes indicated by the data.

Even a casual glance at Fig. 3 assures the reader that Tinsley's $(1972,1977)$ conclusions are basically correct; at redshifts where the cosmological leverage becomes fairly large, the evolutionary effects due to possible different histories of early galactic star formation become much larger. Model details, such as the exact shape of the giant branch and the stellar luminosity function slope near $M=1 M_{\odot}$ are of a minor significance. What matters at large redshifts in the visible regime is the length of any star formation burst, and to some extent, the epoch of the last strong burst (that is, the $z_{G F}$ ) in these giant galaxies. Other data, mainly relevant to non-radio galaxies (see Hamilton 1985, Spinrad 1986) suggests $z_{G F} \geq 4$ for many large $\mathrm{gE}$ galaxies, and so we use $z_{G F}=5$ as a convenient guess value for the models.

The main conclusion of DSD is that the size of the detected evolutionary effects, and the variety of plausible evolutionary histories which affect the emitted UV spectra of galaxies are large at $z>1$, and prevent us from making any firm conclusions about cosmology. Thus, the data and models do not allow sharp cosmological decisions in the visual region. We can say that completely passively evolving galaxies (no large starbursts after the initial one) are rare in our sample of 3CR galaxies, but we will mention a few 3CR candidates for quiet evolution in the Section IV below. This is consistent with O'Connell's (1986) conclusions from population synthesis of nearby $\mathrm{E}$ galaxies. An important future project will be to select optically a sample of very distant clusters $(z \geq 1)$ to complement the 3CR data set shown here. We conclude that these luminous 3CR galaxies are characterized by $\mu$-models with $e$-folding times of $\sim 1-1.5 \mathrm{Gyr}$; such models can also provide enough ionizing radiation (UV starlight) at $\lambda<912 \AA$ to produce the observed emission line intensities, even the far-UV lines (e.g., Ly- $\alpha$ or the carbon lines) in 3C 256 and 3C $239(z \sim 1.8)$. The differences between the passive $c$-models and the more active $\mu$-models fade as the galaxies age, so that at $z \leq 0.5$ no substantive differences remain. In summary, a separation of cosmology and galaxy evolution is quite difficult in the observed $B V R$ bands.

\section{The IR Hubble Diagram: A Chance to Determine $q_{0}$ ?}

As previously stated, the $2 \mu \mathrm{m}$ photometry samples mainly the light from old stars, and thus it should be much less sensitive to the fraction of young stars in these distant galaxies. The photometry of $3 \mathrm{CR}$ sources by LL and LE contains a sufficient overlap to permit a comparison of magnitudes for 5 radio galaxies with $K>15$; the photometric agreement is good with a slight zero point offset. We have corrected the LL and Lilly et al. (1985b) $K$-band data by $-0^{m} .15$ to place everything on the LE system. There have been no aperture, richness, or $\mathrm{K}$-corrections to any of the data; these corrections were applied to the models instead. 
Figure 4 shows a $K$-band Hubble diagram, and some of the Bruzual evolutionary models. In this and the other figures showing the $K$-band Hubble diagrams, we have normalized the models at small $(z<0.15)$ redshifts; aperture corrections were applied for that purpose, but are not shown on the plotted data points. This Figure illustrates the lack of sensitivity to the galaxy formation redshift, $z_{G F}$. This insensitivity to evolutionary variables is typical for the models in the near-IR, as opposed to our conclusions from the $V$ band, where the galaxy evolution dominated the Hubble diagram and the conclusions drawn from it. In the $K$-band we mainly see the mild evolution of the red giant branch, and the relatively slow-changing $\mathrm{K}$-corrections.

The models in the near-IR are also insensitive to the SFR history: whether the SFR is prolonged or in a single burst. Figure 5 shows all currently available $K$-band photometry for distant galaxies, compared to the same $c$ and $\mu$ models shown for our $V$-band Hubble diagram of Fig. 3. The reader should note that at $2 \mu \mathrm{m}$ the evolutionary model differences are very small, and there is not much sensitivity to the adopted $H_{0}$, which enters in the conversion of model timesteps to redshifts. The evolutionary differences are now overshadowed by the cosmological curvature differences between open and closed world models. This may be the cosmologically sensitive type of diagram that many investigators have long hoped for; a choice between global geometries should be possible from data of this quality at redshifts this large. Recall that the previous investigations often showed the scatter of galaxy magnitudes in excess of the differences between decelerations of

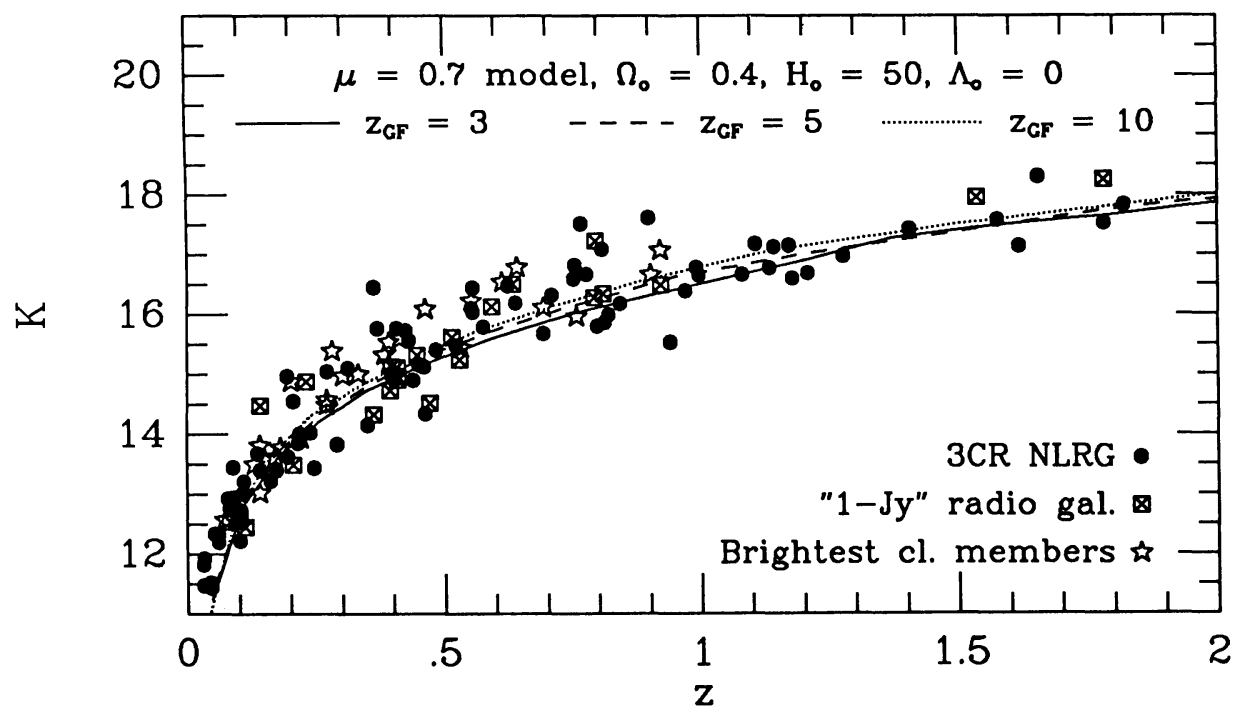

Figure 4. An infrared $K$-band Hubble diagram, used to illustrate the insensitivity to evolution models at long emitted wavelengths, and in particular the insensitivity to the redshift of galaxy formation, $z_{G F}$. The data points are BCM's from LE, the "1 Jy" radio galaxies of Allington-Smith et al., and the narrow-line 3CR galaxies of LL and LE. The data are quite homogenous, and no corrections of any kind have been applied. Thus, at $z \leqslant 0.1$, the models appear slightly too bright $(K \leq 13)$ because the necessary aperture corrections to the galaxy photometry have not been explicitly applied to the data. 
$q_{0}=0$ and $q_{0}=1$. The 3CR galaxies have a fairly small luminosity dispersion in the near-IR, roughly $\sigma(m) \simeq 0.5$, independent of the redshift.

But before we feel too optimistic about the deceleration parameter which the eye would chose in Figure $5\left(q_{0} \simeq 0.25\right.$, say), we ought to consider any possible sources of systematic errors. Hardest of all is the evaluation of the quality and appropriateness of the red giant branch (RGB) spectra adopted by Bruzual (1983), and the post-RGB evolution ( $c f$. Renzini and Buzzoni 1986). All we can do empirically is to note that the predicted broad-band colors, $(V-K)$ of the models, which are sensitive to the giant branch shape and the ratio of main sequence stars and subgiants to the luminous giants, fit the available data at $z \leq 0.45$ quite well (Bruzual 1983, 1986). This is a fair, but obviously an incomplete check. New models are needed, with improved post-RGB tracks, and with a more complete library of stellar spectra, including more metal-rich giants; that work could take some time, and is obviously beyond the scope of this review.

The second worry is the quality of our three types of "standard candles" in

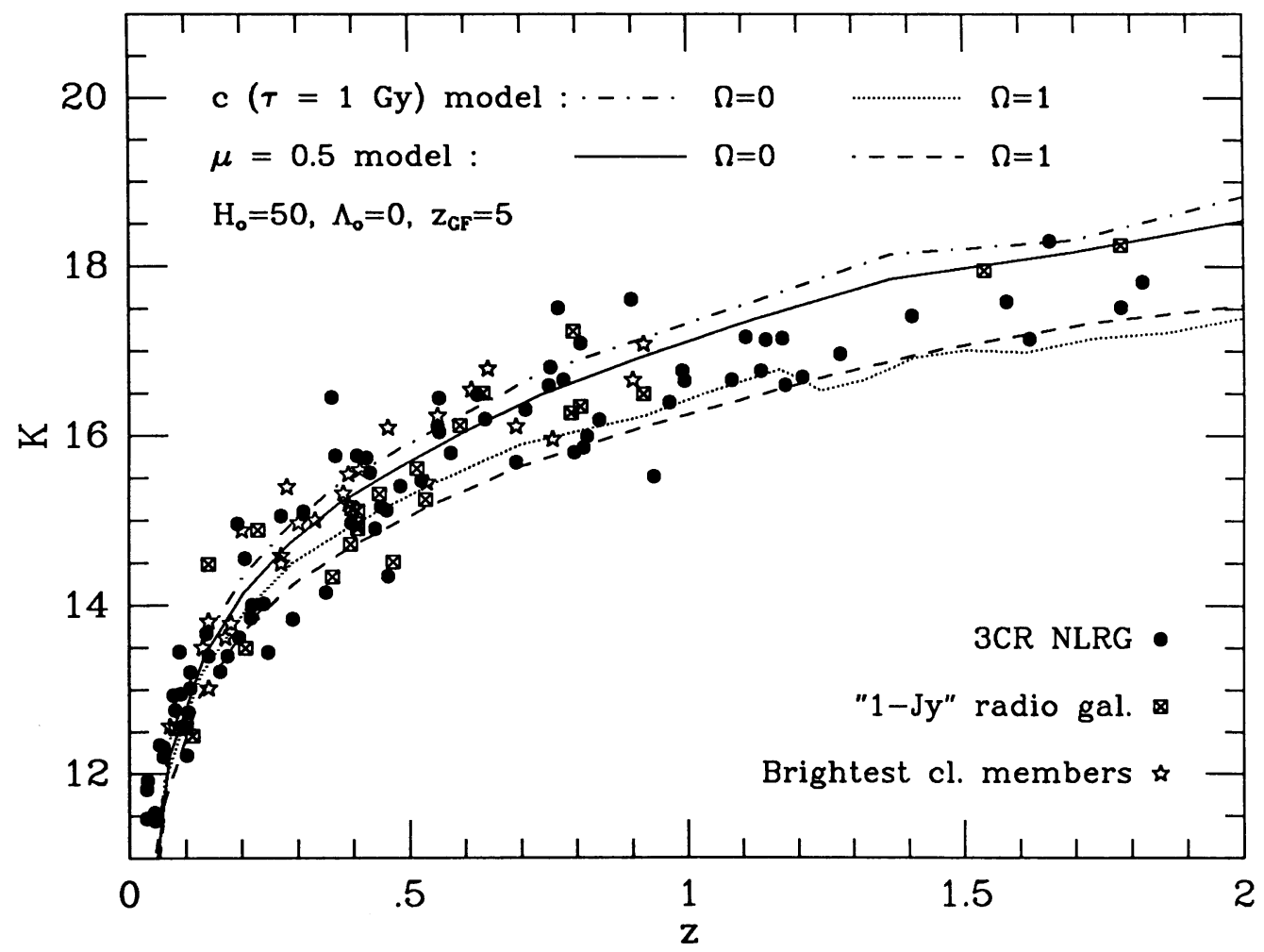

Figure 5. The complete $K$-band photometry sample is compared to the Bruzual evolution and standard cosmology models shown before in Fig. 3. There is now a good sensitivity to cosmological parameters. The $q_{0}$ probably lies between 0.2 and 0.3 , judging from this data set. The models are also quite insensitive to the adopted $H_{0}$. Galaxy evolution differences here are much less important than what we see in the visual region. 
the near-IR. A careful comparison of the $K$-band luminosities of the BCM's $0.05 \leq$ $z \leq 0.92)$, "1 $\mathrm{Jy}$ " radio galaxies $(0.1 \leq z \leq 1.78)$, and the $3 \mathrm{CR}$ galaxies $(0.04 \leq$ $z \leq 1.82)$ in their overlap regions suggests that at small redshifts $(z<0.4)$ the first-ranked cluster gE's are about $0^{m} .3 \pm 0^{m} .2$ fainter than the $3 \mathrm{CR}$ galaxies; the "1 Jy" galaxies have about the same $M_{K}$ as the 3CR galaxies at low redshifts, -26 or so for $H_{0}=50 \mathrm{~km} \mathrm{~s}^{-1} \mathrm{Mpc}^{-1}$. However, at $z>1$, the two available "1 Jy" galaxies are slightly fainter $\left(\leq 0^{m} .4\right)$ than $3 \mathrm{CR}$ galaxies with the similar redshifts. Can these zero-point $M_{K}$ differences sway our world-model conclusions? Not entirely, since $\Delta q_{0}=0.5$ corresponds to $\Delta K \approx 1^{m}$ at $1<z<1.8$. Any possible luminosity differences which are systematic with redshift can be much more serious. Recently Yates, Miller, and Peacock (1986) have suggested that the most powerful 3CR galaxies, at $z \geq 1$, are more luminous than the lower redshift 3CR's and the " 1 Jy" galaxies at the highest redshifts. A correlation with radio power and optical luminosity cannot be pervasive in our samples, since the "1 $\mathrm{Jy}$ " and $\mathrm{BCM}$ luminosities are virtually identical in $M_{K}$ at $z \sim 0.8$, and their radio powers are vastly different at those redshifts. But if we restrict our comparison to the most powerful radio galaxies with $P_{178}>10^{27} W \mathrm{~Hz}^{-1} \mathrm{sr}^{-1}$, a correlation between $M_{K}$ and $P_{178}$ may well be present. Recall that at $z>1$, the "1 Jy" galaxies appear slightly fainter than 3CR galaxies shown in Fig. 5. However, we do not find any correlation between cluster membership and radio power. To explore the tentative Yates et al. correlation somewhat further, we have tried to put together an IR Hubble diagram at approximately equal radio power $(\log P \sim 27)$, using the highflux 3CR sample at $z<0.5$ and the " $1 \mathrm{Jy}$ " sample at $z>0.7$. Figure 6 shows this composite diagram. The model line for $q_{0} \simeq+0.2$ is a good fit to this data set. Using only the 3CR data set, we obtain a best fit with $q_{0} \simeq+0.5$; these differences may be caused in part by the data systematics.

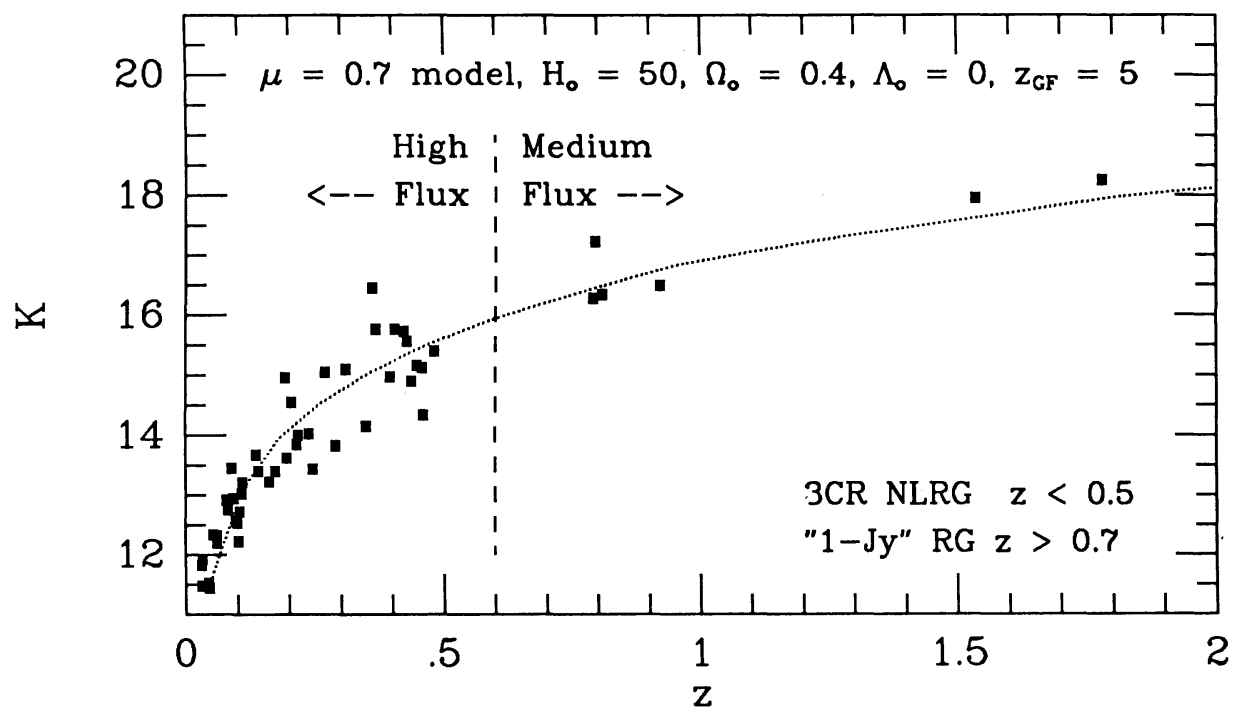

Figure 6. An infrared Hubble diagram combining the data points from the $3 \mathrm{CR}$ set $(z<0.5)$ and the " $1 \mathrm{Jy}$ " galaxies $(z>0.70)$. The idea is to equalize roughly the radio power at all observed cosmic epochs; the effect is to decrease slightly $q_{0}$ - our model is now quite satisfactory for $q_{0}=+0.2, \Lambda_{0}=0$. This probably is a very sensible compromise solution. 
Finally, there is another possible bias: if galaxy mergers or cannibalism are important in the early formation of giant elliptical galaxies (especially in protoclusters) as originally suggested by Ostriker and Hausmann (1977), and more recently by Davis (1986) from N-body simulations, then our $q_{0}$ (apparent) will be a systematic underestimate of $q_{0}$ (true). This is because $\mathrm{gE}$ or $\mathrm{cD}$ galaxies become brighter as they age. But recall that most of the weight of our samples is at $z>0.7$, where $90 \%$ of the galaxies which we use are strong radio emitters. Considerable recent work in this field has indicated a qualitative connection between the presence of radio emission and galaxy interactions. These $3 \mathrm{CR}$ and " 1 Jy" galaxies are, then, the systems that evolved dynamically very early on, and they may have completed their merger growth at the epoch we observe them.

So, it appears that little or no systematic correction is needed in the comparison of $z<0.8$ galaxies with their distant counterparts. Thus, we conclude by suggesting a value of $q_{0}=0.2 \pm 0.3$ as a best guess from the 1986 Hubble diagrams in the $2 \mu \mathrm{m}$ region. This is an estimate, rather than a measurement, but we believe that this value of $q_{0}$ is more reliable than the previous measurements using similar methods, and it is consistent with other modern estimates of the density parameter $\Omega_{0}$ (Peebles 1986).

\section{Consistency Checks Over the Whole Spectrum}

It is possible to use the visual and the near-IR color and magnitude data together to check whether the same $\mu$-models/cosmology combinations are indicated over the entire available wavelength range. Following LL, we note that the $(R-K)$ color provides our best broad-brush coverage of the UV-optical spectrum, but by itself it cannot constrain the $q_{0}$ or the evolution models very well. If we adopt $q_{0} \simeq 0.25$, we would conclude, as do LL, that the galaxies mainly follow a $\mu$-model, but some are sufficiently faint and red as to suggest a passive $c$-model evolutionary history. Among these distant red galaxies are 3C 65, 3C 68.2, and 3C 241. Their mean redshift is $\langle z\rangle=1.45$. The data in the visual regime indicate the more active models, $\mu \sim 0.6$, but the data in near-IR are consistent with the quieter models, $\mu \sim 0.8$, or even the passive $c$-models. The interpretation of this moderate discrepancy is unclear; it could be due to some unknown model flaw, but it can also be understood simply by assuming a two-component population model.

This scenario suggests that the majority of the stars are fairly old, but a small fraction of the mass is now undergoing a starburst (associated also with the presence of a radio source?). That would make the composite starlight at short wavelengths resemble a $\mu$-model (decaying SFR) for a few Gyr. A sequence of such sporadic starbursts declining in time is what the $\mu$-models were intended to represent anyway. On the other hand, the few faint and red galaxies may have lead a quiet existence since their initial collapse. A future check on this "color accord problem" could come from new data on more distant cluster or " $1 \mathrm{Jy}$ " red galaxies at large redshifts. At the moment the inconsistency in our derived $\mu$ as a function of wavelength leads to only some mild doubts about our quantitative application, and should not affect our $q_{0}$ measurements appreciably. We note also that the correlation of "UV-excess" with the radio galaxy emission line strengths has weakened as more spectroscopic data have become available. 


\section{Desiderata for the Future, and the Concluding Remarks}

The progress of the 1980's is very encouraging, and we expect the field to advance by extension of observations on these three types of standard candles toward even greater lookback times. We listed some of the possible problems which need more attention, but new problems will arise as well. For example, what is the effective formation epoch of clusters? Will their initial density contrast be a major factor (and a bias) in their collapse time and later recognition, by either optical or X-ray techniques?

There is hope that some of the " $1 \mathrm{Jy}$ " radio galaxies whose observations have just really begun, may prove to be very distant and secure cosmological probes, perhaps at $z \leq 2$, if the $(K, z)$ relations we show here may be extrapolated. Future imaging photometry in the near-IR will greatly improve the quality of the raw $K$ photometric data at faint levels ( $K \geq 18$, say), and we look forward to that capability. Finally, some independent method to check the validity of evolutionary models has to be established. Then we should be able to capitalize on the distant galaxy data sets like those presented here in attacking one of the cosmology's oldest and most important problems.

We thank Drs. M. Rieke, J. Silk, R. Wyse, and M. Davis for their comments and encouragement, and for the use of unpublished data. Mark Dickinson provided valuable assistance with our new CCD photometry, and Patrick McCarthy and Michael Strauss helped with related work. H. S. wishes to acknowledge continued support form the U.S. National Science Foundation, and S. D. acknowledges partial support from Harvard University. We again thank the staffs of Kitt Peak, Lick, MMT, and CFHT observatories for their assistance in gathering much of the observational data presented here.

\section{REFERENCES:}

Allington-Smith, J. R., Lilly, S. J., and Longair, M.S. 1985, M.N.R.A.S. 213, 243. Bruzual, G. 1983, Ap.J. 273, 105.

Bruzual, G. 1986, in Spectral Evolution of Galaxies, C. Chiosi and A. Renzini (eds.), p. 263. Dordrecht: D. Reidel.

Davis, M. 1986, private communicaton.

Djorgovski, S., Spinrad, H., and Dickinson, M. 1987, Ap.J. (in press). [DSD]

Djorgovski, S., Spinrad, H., and Marr, J. 1985, in New Aspects of Galaxy Photometry, J.L. Nieto (ed.), p. 193. New York: Springer Verlag.

Grasdalen, G. 1980, in Objects of High Redshift, IAU Symposium \#92, G. O. Abell and P. J. E. Peebles (eds.), p. 269. Dordrecht: D. Reidel.

Gunn, J. E., and Oke, J. B., 1975, Ap.J. 195, 255.

Gunn, J. E., Hoessel, J., and Oke, J. B. 1986, Ap.J. 306, 30.

Hamilton, D. 1985, Ap.J. 297, 371.

Hoessel, J., Gunn, J. E., and Thuan, T. X. 1980, Ap.J. 241, 486.

Kristian, J., Sandage, A. R., and Westphal, J. 1978, Ap.J. 221, 383.

Lebofsky, M. 1980 in Objects of High Redshift IAU Symposium \#92, G. O. Abell and P. J. E. Peebles (eds.), p. 257. Dordrecht: D. Reidel.

Lebofsky, M. J. 1981 Ap.J. 245, L59.

Lebofsky, M. J., and Eisenhardt, P. R. M. 1986, Ap.J. 300, 151. [LE] 
Lilly, S. J., and Longair, M. S. 1982, M.N.R.A.S. 199, 1053. [LL]

Lilly, S. J. 1983, Ph. D. Thesis, University of Edinburgh, UK.

Lilly, S. J., and Longair, M. S. 1984, M.N.R.A.S. 211, 833. [LL]

Lilly, S. J., Longair, M. S., and Miller, L. 1985a, M.N.R.A.S. 214, 109.

Lilly, S. J., Longair, M. S., and Allington-Smith, J. R. 1985b, M.N.R.A.S. 215, 37.

O'Connell, R. 1986, to appear in STScI Conference on Stellar Populations, M. Tosi and C. Norman (eds.), Cambridge University Press.

Ostriker, J., and Hausman, M. 1977, Ap.J. 217, L125.

Peebles, P. J. E. 1986, Nature 321, 27.

Perryman, M. A. C., Lilly, S. J., Longair, M. S., and Downes, A. J. B. 1984, M.N.R.A.S. 209, 159.

Renzini, A., and Buzzoni, A. 1986 in Spectral Evolution of Galaxies, C. Chiosi and A. Renzini (eds.), p. 195. Dordrecht: D. Reidel.

Sandage, A. R. 1972, Ap.J. 178, 1.

Sandage, A. R. 1975, in "Galaxies and the Universe", Vol. IX of Stars and Stellar Systems, A. Sandage, M. Sandage, and J. Kristian (eds.), p. 761. Chicago: University of Chicago Press.

Sandage, A. R., Kristian, J., and Westphal, J. A. 1976, Ap.J. 205, 688.

Schneider, D. P., Gunn, J. E., and Hoessel, J. B. 1983, Ap.J. 264, 337.

Spinrad, H. 1986, Publ. Astr. Soc. Pacific 98, 269.

Spinrad, H., and Djorgovski, S. 1984, Ap.J. 285, L49.

Spinrad, H., Djorgovski, S., Marr, J., and Aguilar, L. 1985, Publ. Astr. Soc. Pacific 97, 932.

Tinsley, B. T. 1972, Ap.J. 173, L93.

Tinsley, B. T. 1977, Ap.J. 211, 621.

Yates, M. G., Miller, L., and Peacock, J. A. 1986, M.N.R.A.S. 221, 311.

\section{DISCUSSION}

MILEY: I would like to report evidence that indicates that the highluminosity radio galaxies are indeed different from the lowerluminosity radio galaxies and that plotting their magnitudes on the same diagram may well introduce selection effects. First, a survey of lower-z matched samples of high-luminosity and low-luminosity radio galaxies by Heckman, Illingworth, van Breugel, Bothun, Baum, Smith and myself shows that the higher powered sources have preferentially peculiar optical morphologies. Second, Golombek, Neugebauer and I have shown that they have IRAS (60-micron) excesses compared with low-luminosity radio galaxies. Both these results suggest that the high-powered radio galaxies are undergoing mergers and are definitely not normal ellipticals as presumed by conventional astronomical folk-lore.

SPINRAD: Yes, there is little doubt that some radio galaxies have disturbed outer isophotes. Whether that would matter in a centeredaperture light measurement that includes the inner 60-80 kpc (D), is, to me, unclear. 
I also believe that the IR is more immme to these "disturbances", if the latter are young-object dominated.

The Hubble-diagrams used here, are, in fact, for very luminous sources - radio galaxies well above the slope-change in the RLF.

WINDHORST: First, an addition to George Miley's comment. At much fainter radio fluxes, milli Jansky levels, the radio source population also consists of normal giant ellipticals and weird looking beasts. So we can always construct a subsample of "normal looking" giant ellipticals for the purpose of a Hubble diagram. My question is twofold. I can imagine two effects that couldd brighten the K-magnitude of galaxies at $z \sim 1$ with respect to the model predictions. First, the l'aschen or other lines might enhance the K-luminosity and second, AGB stars or carbon stars might produce more light at $\lambda \geqslant 1 \mu$. I am sure you share this worry, but could you give us some feeling how important these effects might be for the Hubble diagram in $\mathrm{K}$.

SPINRAD: I doubt if any infrared emission lines would compete with the stellar energy maxima and the very broad-band IR spectral response(s). Emission lines should hardly modulate $\underline{\mathrm{K}}$ magnitudes.

The giant branch details now follow the Yale evolutionary tracks. The current Bruzual models are a satisfactory match in color $(\mathrm{V}-\mathrm{K})$ for redshifts $0<z \leq 0.5$; this gives me some limited confidence that the stellar giant branch is fairly realistic for recent cosmic epochs. At longer look-backs (e.g. - younger turnoffs and more-massive giant precursor stars), I cannot make a comparison of theory with the E galaxy data. So the question will have to be answered indirectly, presumably by tests Dr. Bruzual is now assembling. Good question!

LONGAIR: It is a great pleasure to have the opportunity to express my admiration for the remarkable achievement which Dr. Spinrad has made in measuring the redshifts and spectra of the 3C and 1-Jy radio galaxies. I believe the studies of the faintest of the radio galaxies in the 1-Jy sample are important for understanding when these galaxies first formed. Simon Lilly, Jeremy Allington-Smith and I showed that, among the faintest and presumably most distant of the galaxies in the 1-Jy sample, there are objects with the optical-infrared colors of passively evolving elliptical galaxies. Since their redshifts are presumably in the range $1.5 \leqslant z \leqslant 3$, this means that they must have formed their stellar populations at redshifts greater than 3.5. The study of these galaxies is very important in setting astrophysical constraints upon when these massive galaxies first formed. 